\title{
Long-term follow-up results of umbilical hernia repair
}

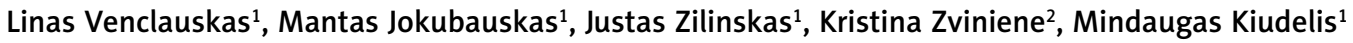 \\ ${ }^{1}$ Department of Surgery, Lithuanian University of Health Sciences, Kaunas, Lithuania \\ ${ }^{2}$ Department of Radiology, Lithuanian University of Health Sciences, Kaunas, Lithuania
}

Videosurgery Miniinv 2017; 12 (4): 350-356

DOI: https://doi.org/10.5114/wiitm.2017.70327

\begin{abstract}
Introduction: Multiple suture techniques and various mesh repairs are used in open or laparoscopic umbilical hernia (UH) surgery.

Aim: To compare long-term follow-up results of UH repair in different hernia surgery groups and to identify risk factors for $U H$ recurrence.

Material and methods: A retrospective analysis of 216 patients who underwent elective surgery for UH during a 10year period was performed. The patients were divided into three groups according to surgery technique (suture, mesh and laparoscopic repair). Early and long-term follow-up results including hospital stay, postoperative general and wound complications, recurrence rate and postoperative patient complaints were reviewed. Risk factors for recurrence were also analyzed.

Results: One hundred and forty-six patients were operated on using suture repair, 52 using open mesh and 18 using laparoscopic repair technique. $77.8 \%$ of patients underwent long-term follow-up. The postoperative wound complication rate and long-term postoperative complaints were significantly higher in the open mesh repair group. The overall hernia recurrence rate was $13.1 \%$. Only $2(1.7 \%)$ patients with small hernias $(<2 \mathrm{~cm})$ had a recurrence in the suture repair group. Logistic regression analysis showed that body mass index $(B M I)>30 \mathrm{~kg} / \mathrm{m}^{2}$, diabetes and wound infection were independent risk factors for umbilical hernia recurrence.

Conclusions: The overall umbilical hernia recurrence rate was $13.1 \%$. Body mass index $>30 \mathrm{~kg} / \mathrm{m}^{2}$, diabetes and wound infection were independent risk factors for $\mathrm{UH}$ recurrence. According to our study results, laparoscopic medium and large umbilical hernia repair has slight advantages over open mesh repair concerning early postoperative complications, long-term postoperative pain and recurrence.
\end{abstract}

Key words: umbilical hernia, recurrence rate, laparoscopic repair.

\section{Introduction}

The incidence of umbilical hernia (UH) varies from $6 \%$ to $14 \%$ of all abdominal wall hernias in adults. Umbilical hernia repair is a common surgical procedure in general surgery [1-4]. Multiple suture techniques and various mesh repairs are used in open $\mathrm{UH}$ surgery while laparoscopic repair is rapidly increasing in large UH. The recurrence rate after suture repair technique ranges from $10 \%$ to $30 \%$ compared with up to $10 \%$ in the mesh repair group [3]. There is no discussion as to which technique (suture or mesh repair) we have to use in elective surgery when the $\mathrm{UH}$ size is $>3 \mathrm{~cm}$. The optimal choice is mesh technique. However, there is much debate involving surgery for small UH $(<2-3 \mathrm{~cm})$. Only a few randomized controlled trials (RCTs) and prospective studies have analyzed long-term UH treatment results [1, 5-9].

Several factors have been responsible for recurrence after umbilical hernia repair. However, only

\section{Address for correspondence}

Mantas Jokubauskas, Department of Surgery, Lithuanian University of Health Sciences, 2 Eiveniu St, 50161 Kaunas, Lithuania,

phone: +37 061852125, e-mail: mantas910317@gmail.com 
a few studies have presented an independent factor after multivariate analysis [10, 11]. Large seroma, surgical site infection, the patient's body mass index $(\mathrm{BMI})>30 \mathrm{~kg} / \mathrm{m}^{2}$ and hernia defects $>2 \mathrm{~cm}$ have been reported as possible factors for umbilical hernia recurrence.

\section{Aim}

To compare long-term follow-up results of UH repair in different hernia surgery groups and to identify risk factors for UH recurrence.

\section{Material and methods}

The institutional review board's permission no. BEC-MF-07 was obtained prior to this study. It was a retrospective study of 216 patients who underwent elective surgery for primary UH. Patients were operated on in the Department of Surgery between $1^{\text {st }}$ January 2002 and $31^{\text {st }}$ December 2012. We tried to contact all treated patients by phone. All contacted patients were invited to attend the physical examination in our outpatient department. Hernia recurrence diagnosis was based on the patient's physical examination and paraumbilical area ultrasound, performed by an experienced radiologist. If the ultrasound examination could not identify hernia recurrence (obese patient or uncertain image), an abdominal wall computed tomography (CT) scan was performed. The patient's age, gender, hospital stay, hernia size, BMI, postoperative general and wound complications, recurrence rate, risk factors for hernia recurrence, postoperative pain and patient's assessment of surgery outcome were analyzed. Postoperative pain was evaluated by patients themselves according to two criteria: any pain or discomfort in the surgery area at rest and during physical activity.

Patients assessed their surgery outcome according to the following evaluation criteria: excellent, very good, good, satisfactory, poor and very poor.

All the patients were divided into three groups according to hernia repair technique: suture repair, mesh repair and laparoscopic repair. Umbilical hernias were divided into three groups based on their size according to the European Hernia Society (EHS) recommendations: small UH $(<2 \mathrm{~cm})$, medium $(2-4 \mathrm{~cm})$ and large $(>4 \mathrm{~cm})[12]$.

\section{Surgical techniques}

The type of UH hernia repair technique was chosen independently by the operating surgeon. The su- ture repair was performed using the Keel technique: the hernia defect was closed using interrupted inverted monofilament slowly absorbable sutures.

The onlay and sublay techniques were used for open mesh repair surgery. The appropriate size Optilene mesh (by B/Braun) was used for both techniques. The mesh was secured with a few non-absorbable sutures on the posterior sheet of aponeurosis in the sublay technique. The mesh was fixed with interrupted, non-absorbable sutures on the superior sheet of aponeurosis in the onlay technique. The need for wound drainage in open mesh repair surgery was chosen independently by the operating surgeon.

Three trocars (one $10 \mathrm{~mm}$ and two $5 \mathrm{~mm}$ in size) were used for laparoscopic hernia repair. Appropriate size Physiomesh (by Johnson \& Johnson) was used for defect closure. The mesh was secured with titanium tacks (ProTack, Covidien). The hernia defect was not closed before the mesh placement. The mesh size in all open and laparoscopic techniques was chosen so that it overlapped by $5 \mathrm{~cm}$ in all directions beyond the outer border of the fascial defect.

\section{Statistical analysis}

Statistical analyses were performed using SPSS Statistics 20.0 for Windows. Student's t-test was used to compare parametric variables. $\chi^{2}$ test and Kruskal-Wallis one-way analysis of variance were used to compare nonparametric variables between groups. Logistic regression analysis was used to assess the risk factors for hernia recurrence. Data were expressed as mean and standard deviation. The value of $p<0.05$ was considered statistically significant.

\section{Results}

Two hundred and sixteen patients underwent elective surgery for UH over a 10-year period. The mean patients' age was $54.3 \pm 15.7$ years. Most of them were female ( $n=137,63.4 \%)$. From 52 patients who underwent open mesh repair, in 39 (75\%) the onlay technique and in only 13 (25\%) patients the sublay technique was used. Patients' general characteristics and distribution between the groups are summarized in Table I.

Patients' BMI was significantly higher in the open mesh repair group compared with open suture ( $p<$ $0.001)$ and laparoscopic repair $(p=0.004)$ groups. Average defect size in the open mesh repair group 
Table I. Patient general characteristics

\begin{tabular}{|c|c|c|c|c|}
\hline Variable & Suture repair & Open mesh repair & Laparoscopic repair & $P$-value \\
\hline$N(\%)$ & $146(67.6)$ & $52(24.1)$ & $18(8.3)$ & - \\
\hline Age [years] & $54.5 \pm 16.8$ & 54.912 .7 & $51.1 \pm 14.3$ & NS \\
\hline \multicolumn{5}{|l|}{ Gender, $n(\%)$ : } \\
\hline Male & 49 (33.6) & $24(46.2)$ & $6(33.3)$ & \multirow{2}{*}{$\begin{array}{c}\text { NS } \\
\text { (between gender gr.) }\end{array}$} \\
\hline Female & $97(66.4)$ & $28(53.8)$ & $12(66.7)$ & \\
\hline Median ASA score (range) & $2(1-3)$ & $2(1-4)$ & $2(1-3)$ & NS \\
\hline Smoking history, $n$ (\%) & $29(19.9)$ & $11(21.2)$ & $3(16.7)$ & NS \\
\hline \multicolumn{5}{|l|}{ Medical history, $n(\%)$ : } \\
\hline Cancer & $3(2.1)$ & $2(3.8)$ & 0 & NS \\
\hline Diabetes & $8(5.5)$ & $4(9.6)$ & $3(16.7)$ & NS \\
\hline Steroid use & $1(0.7)$ & $1(1.9)$ & 0 & NS \\
\hline Other hernia & $4(2.7)$ & $2(3.8)$ & 0 & NS \\
\hline BMI $\left[\mathrm{kg} / \mathrm{m}^{2}\right]$ & $30.4 \pm 7.0$ & $36 \pm 6.9$ & $28.9 \pm 6.6$ & $\begin{array}{l}<0.001 \\
\text { NS } \\
0.004\end{array}$ \\
\hline Umbilical hernia size [cm] & $2.69 \pm 1.21$ & $4.64 \pm 4.36$ & $3.12 \pm 1.22$ & $\begin{array}{c}<0.001 \\
\text { NS } \\
0.024\end{array}$ \\
\hline
\end{tabular}

was significantly larger than in the suture $(p<0.001)$ and laparoscopic repair ( $p=0.024$ ) groups (Table I). Mesh repair technique was used significantly more often when the hernia defect was $>4 \mathrm{~cm}(p=0.001)$. The relation between hernia repair technique and hernia defect size is demonstrated in Table II.

One hundred and sixty-eight (77.8\%) patients attended our outpatient clinic in the late follow-up period and were examined during their outpatient visit: 115 patients - suture repair group, 38 patients - open mesh repair group (30 patients - onlay, 8 patients - sublay) and 15 patients - laparoscopic repair group. Median follow-up time was
56 months with no significant difference between the groups. Forty-eight (22.2\%) patients were lost to follow-up: 4 patients changed their residence with no forwarding address present, 2 patients refused to participate in the study and 42 patients gave no response.

The overall postoperative complication rate was $12 \%$ in the early follow-up period. This rate was higher in the open mesh repair group than in the laparoscopic and suture repair groups (26.9\% vs. $11 \%$ vs. $6.8 \%$; $p>0.05$ ). Most of the complications occurred in patients with hernia defect size $>4 \mathrm{~cm}$, but with no significant difference.

Table II. Relation between hernia repair technique and hernia size

\begin{tabular}{|lccc|}
\hline Surgery technique & \multicolumn{1}{c|}{ Hernia size $[\mathrm{cm}]$} & $>4$ \\
\cline { 2 - 4 } & $<2$ & $2-4$ & $15(10.3 \%)$ \\
\hline Suture repair (146) & $28(19.2 \%)$ & $103(70.5 \%)$ & $23(44.2 \%)$ \\
\hline Mesh repair (52) & $4(7.7 \%)$ & $25(48.1 \%)$ & $4(22.2 \%)$ \\
\hline Laparoscopic repair (18) & $2(11.1 \%)$ & $12(66.7 \%)$ & $42(19.4 \%)$ \\
\hline Total (216) & $34(15.8 \%)$ & $140(64.8 \%)$ & 4 \\
\hline
\end{tabular}


Four patients in the open mesh group and $1 \mathrm{pa}-$ tient in the suture group had a subcutaneous fistula which existed up to 3 weeks after surgery (Table III) and closed spontaneously after some time.

Long-term follow-up results demonstrated that pain or discomfort in the umbilical region was more often present in the open mesh repair group as well as at rest or during physical activity (Table IV) compared with suture and laparoscopic repair groups.

About half of all patients (47-67\%) assessed the surgery outcome as excellent or very good, while only a few of them (2-6\%) assessed it as poor or very poor, without any significant difference between the groups (Table IV).
The overall umbilical hernia recurrence rate was $13.1 \%(n=22)$ in our study. The recurrence rate was slightly higher in the open mesh repair group with no significant difference (Table IV). Only 2 (1.7\%) patients with small hernias $(<2 \mathrm{~cm})$ had hernia recurrence in the suture repair group. The recurrence rate was significantly higher in patients whose BMI was $>30 \mathrm{~kg} / \mathrm{m}^{2}(p=0.026)$. The average $\mathrm{BMI}$ of patients with recurrence was $34.9 \pm 6.6$ vs. $31.8 \pm 7.2$ in patients who had no hernia recurrence $(p=0.037)$. We did not find any significant difference in recurrence rate between different hernia size groups.

Logistic regression analysis showed that only $\mathrm{BMI}>30 \mathrm{~kg} / \mathrm{m}^{2}$ and $\mathrm{BMI}>30 \mathrm{~kg} / \mathrm{m}^{2}$ with diabetes,

Table III. Early follow-up results (up to 2 weeks after surgery)

\begin{tabular}{|c|c|c|c|c|}
\hline Variable & $\begin{array}{l}\text { Suture } \\
(n=146)\end{array}$ & $\begin{array}{c}\text { Mesh } \\
(n=52)\end{array}$ & $\begin{array}{l}\text { Laparoscopic } \\
\quad(n=18)\end{array}$ & $P$-value \\
\hline Operating time [min] & $68.6 \pm 34.1$ & $107.9 \pm 55.7$ & $87.9 \pm 55.5$ & $\begin{array}{c}<0.001 \\
\text { NS } \\
\text { NS }\end{array}$ \\
\hline $\begin{array}{l}\text { Postop. hospital stay } \\
\text { [days] }\end{array}$ & $3.1 \pm 2.2$ & $5.4 \pm 3.3$ & $2.7 \pm 1.6$ & $\begin{array}{l}<0.001 \\
\quad N S \\
<0.001\end{array}$ \\
\hline Seroma & $5(3.4 \%)$ & $4(7.7 \%)$ & $1(5.5 \%)$ & NS \\
\hline Hematoma & $2(1.4 \%)$ & $1(1.9 \%)$ & 0 & NS \\
\hline Wound infection & $2(1.4 \%)$ & $5(9.6 \%)$ & $1(5.5 \%)$ & $\begin{array}{c}<0.05 \\
\text { NS } \\
\text { NS }\end{array}$ \\
\hline Subcutaneous fistula & $1(0.7 \%)$ & $4(7.7 \%)$ & 0 & NS \\
\hline
\end{tabular}

Table IV. Long-term follow-up results

\begin{tabular}{|c|c|c|c|c|}
\hline Variable & $\begin{array}{l}\text { Suture } \\
(n=115)\end{array}$ & $\begin{array}{c}\text { Mesh } \\
(n=38)\end{array}$ & $\begin{array}{l}\text { Laparoscopic } \\
\quad(n=15)\end{array}$ & $P$-value \\
\hline $\begin{array}{l}\text { Median follow-up time } \\
\text { [months] }\end{array}$ & $58.0(21-151)$ & $59.5(21-139)$ & $56.5(21-103)$ & NS \\
\hline \multicolumn{5}{|l|}{ Patient evaluation: } \\
\hline Excellent/very good & 77 (67.0\%) & $17(44.7 \%)$ & $7(46.7 \%)$ & \\
\hline Good/ satisfactory & $36(31.3 \%)$ & $20(52.6 \%)$ & $7(46.7 \%)$ & NS \\
\hline Poor/very poor & $2(1.7 \%)$ & $1(2.7 \%)$ & $1(6.6 \%)$ & \\
\hline Pain at rest & $5(4.3 \%)$ & $6(15.9 \%)$ & 0 & $\begin{array}{c}0.027 \\
\text { NS } \\
0.039\end{array}$ \\
\hline $\begin{array}{l}\text { Pain during physical } \\
\text { activity }\end{array}$ & $27(23.5 \%)$ & $18(47.4 \%)$ & $3(20 \%)$ & $\begin{array}{c}0.018 \\
\text { NS } \\
\text { NS }\end{array}$ \\
\hline Recurrence & $14(12.2 \%)$ & $7(18.4 \%)$ & $1(6.7 \%)$ & NS \\
\hline
\end{tabular}


Table V. Risk factors for umbilical hernia recurrence

\begin{tabular}{|lccc|}
\hline Factor & Odds ratio & $95 \% \mathrm{Cl}$ & $P$-value \\
\hline $\mathrm{BMI}>30 \mathrm{~kg} / \mathrm{m}^{2}$ & 3.54 & $1.10-11.35$ & 0.034 \\
\hline $\mathrm{BMI}>30 \mathrm{~kg} / \mathrm{m}^{2}$ and diabetes & 3.59 & $1.11-11.67$ & 0.033 \\
\hline $\mathrm{BMI}>30 \mathrm{~kg} / \mathrm{m}^{2}$ and wound infection & 3.67 & $1.13-11.89$ & 0.03 \\
\hline $\mathrm{BMI}>30 \mathrm{~kg} / \mathrm{m}^{2}$ and wound seroma & 3.58 & $1.12-11.51$ & 0.032 \\
\hline Hernia size $>2$ & 2.59 & $0.33-20.69$ & 0.369 \\
\hline ASA $>2$ & 3.60 & $0.99-13.13$ & 0.052 \\
\hline Cancer & 2.72 & $0.51-14.56$ & 0.242 \\
\hline Diabetes & 1.59 & $0.17-14.64$ & 0.682 \\
\hline Age $>60$ years & 1.60 & $1.10-2.33$ & 0.051 \\
\hline
\end{tabular}

Logistic regression analysis.

wound seroma or wound infection are independent risk factors for UH recurrence. All analyzed risk factors are presented in Table $\mathrm{V}$.

\section{Discussion}

There are four possible locations for mesh placement in umbilical hernia repair: preaponeurotic (onlay technique), on the fascial layer (fixed to the ring), in the retromuscular space (sublay technique) or on the preperitoneal space [13]. In our study we analyzed the long-term follow-up results of the three most popular umbilical hernia operating techniques (Keel, open mesh repair and laparoscopic).

In a randomized, controlled study, conducted by Arroyo et al. [6], the umbilical hernia recurrence rate after suture repair was $11 \%$ and was significantly higher than after mesh repair (1\%). The analysis of the Denmark Nationwide Registry specified [3] that the combined cumulated recurrence rate after 55 months including both mesh and sutured repairs was $18 \%$. They also stated that for sutured repair, the cumulative recurrence rate after 55 months was 21\% (9\% reoperations for recurrence and $12 \%$ clinical recurrences) and $10 \%$ for mesh repair (3\% reoperation for recurrence and $7 \%$ clinical recurrence) $(p=0.001)$. In our study, the overall recurrence rate after 56 months including open mesh, laparoscopic and suture repairs was $13.1 \%$. This is an acceptable number, which does not differ from the data published in the literature. However, we had only a $1.7 \%$ recurrence rate for small hernias $(<2 \mathrm{~cm})$ in the suture repair group. The Denmark Nationwide Registry showed a significantly higher recurrence rate $(p=0.001)$ for small hernias in the suture repair group (5.6\%) compared with the open mesh repair group (2.2\%) [2]. The recurrence rate after open mesh (onlay and sublay) umbilical hernia repair was $18.4 \%$ in our study. Of course, it is striking to observe such a high rate of recurrence, which markedly differs from the numbers $(1-10 \%)$ published in literature $[1-3,6,14]$. It is difficult to explain such results. First of all, this is a retrospective study, which has its limitations in design, and this should be a matter of concern. The patients in this study were not randomized, and the type of operation was chosen by the operating surgeon independently. Concerning this, $90 \%$ of patients in the suture repair group had small or medium umbilical hernias and, conversely, $91 \%$ of patients in the open mesh repair group had medium or large umbilical hernias (these patients' average BMI was more than $36 \mathrm{~kg} / \mathrm{m}^{2}$ ) - these were factors which may have influenced the results of hernia recurrence. Another possible bias in this study is the "learning curve" issue. Earlier, our group of surgeons mainly used the Keel technique as well as the onlay technique in umbilical hernia repair. Therefore, the results in these groups might be superior to those of the sublay and especially the laparoscopic group, a technique with which the authors were not familiar.

There is one more limitation - the power of this retrospective study is insufficient, with low numbers in both the open mesh and especially the laparoscopic arms, and therefore the results should be interpreted with caution.

We had only 13 patients in the open mesh repair sublay technique group in our study, so we could not 
reasonably identify the most appropriate open mesh technique for umbilical hernia repair. On the other hand, the Denmark Nationwide Registry obtained similar recurrence rate results comparing onlay $(2.2 \%)$ vs. sublay $(2.5 \%)$ techniques [2]. Berrevoet et al. observed a higher recurrence rate after intraperitoneal mesh placement $(8.3 \%)$ compared with retromuscular mesh repair (3.6\%) but with no significant difference [1]. We need more RCTs with low risk of bias and a higher level of evidence to determine the most appropriate open surgery technique for umbilical hernia. One of them may be the continuous MORPHEUS study (RCT) from the Netherlands, which compares different open mesh repair techniques for small umbilical and epigastric hernias [15].

The recurrence rate after laparoscopic hernia repair in our study was $6.7 \%$. On the other hand, only one patient developed hernia recurrence in the laparoscopic repair group, and the recurrence rate is relatively high due to the small number of patients in this group. Eriksen et al. [8] in their controlled double-blinded study with 17 patients in each arm also found a $6 \%$ recurrence rate after laparoscopic umbilical hernia repair in the tack group, and even a 17\% recurrence rate in the fibrin sealant group.

Despite its popularity, laparoscopic umbilical hernia repair continues to have recurrence. Several surgeons prefer closure of the abdominal wall defect with nonabsorbable sutures prior to the placement of the mesh in selected cases. This technical modification is guided by the notion that appropriate fascial edge approximation might ensure satisfactory overlap of the defect with the mesh, even in the event of mesh shrinkage. Banerjee et al. [16] in their retrospective study compared the postoperative results of two laparoscopic techniques (only mesh placement vs. primary repair with mesh placement) for epigastric, parastomal, umbilical and incisional hernia treatment. They results demonstrated that the rate of recurrence in those treated with laparoscopic primary suture repair + mesh underlay was $3 \%$ in comparison with $4.8 \%$ associated with laparoscopic mesh alone $(p=0.54)$ [16]. There were no recurrences in either surgical technique group for primary umbilical hernia patients - the authors did not find any advantage of defect closure prior to mesh placement in laparoscopic umbilical hernia surgery. We did not close the defect in laparoscopic umbilical hernia repair, but we used a sufficient mesh size the overlap of $5 \mathrm{~cm}$ was achieved in all directions beyond the outer border of the fascial defect. On the other hand, we had only one patient with infected seroma in the laparoscopic repair group.

The complication rate in our study was higher in open mesh repair and large hernia $(>4 \mathrm{~cm}$ ) groups. One reasonable explanation is that almost all large umbilical hernias were operated on using the open mesh (onlay) repair technique, and this was the main reason for postoperative wound seroma and infection. Secondly, we had significantly more postoperative complaints after the open mesh repair technique. In our opinion, the main reason for pain is also the onlay repair technique. The non-absorbable sutures were used to fix the mesh on the superior sheet of aponeurosis in this technique, and this factor could be the main cause of postoperative pain and remaining complaints. However, the retrospective study of Erritzoe-Jervild et al. [9] reported that $10 \%$ of patients had moderate or severe pain or discomfort with no hernia recurrence and $31 \%$ of patients with hernia recurrence. The most frequent causes of pain or discomfort were in onlay and suture repair groups compared with the intraperitoneal mesh group [9].

More than $95 \%$ of patients were satisfied with the operation results after a 56-month follow-up period with no significant difference between the groups.

We also tried to identify the independent risk factors for umbilical hernia recurrence. Based on the meta-analysis on primary umbilical hernias performed by Aslani et al. [17], mesh repair patients have a ten-fold decreased recurrence rate compared to suture repair patients. Eriksen et al. [8] found umbilical hernia size to be an important risk factor for recurrence. The Denmark Nationwide Registry [3] stated that sutured repair, patients younger than 52 years, smoking and surgical site infection were independent risk factors for recurrence. Other authors reported smoking, cirrhosis and ascites as independent risk factors for umbilical hernia recurrence [11].

The results of our study, in which the number of cases is considerably smaller, demonstrated patient's BMI > $30 \mathrm{~kg} / \mathrm{m}^{2}$ with diabetes or wound sero$\mathrm{ma}$, and $\mathrm{BMI}>30 \mathrm{~kg} / \mathrm{m}^{2}$ with surgical site infection to be independent risk factors for umbilical hernia recurrence. We did not find the umbilical hernia size to be an independent risk factor for hernia recurrence, although the recurrence rate was slightly higher with hernia size $>2 \mathrm{~cm}$. 


\section{Conclusions}

The overall umbilical hernia recurrence rate was $13.1 \%$ in our study. Body mass index $>30 \mathrm{~kg} / \mathrm{m}^{2}$, diabetes and wound infection were independent risk factors for UH recurrence. According to our study results, laparoscopic medium umbilical hernia repair has slight advantages over open mesh repair concerning early postoperative complications, long-term postoperative pain and recurrence. Which technique (open suture or mesh repair) is acceptable for small $(<2 \mathrm{~cm})$ and (open mesh or laparoscopic) for large ( $>4 \mathrm{~cm}$ ) umbilical hernias is still the subject of discussions. We need more randomized controlled trials to find the answer.

\section{Conflict of interest}

The authors declare no conflict of interest.

\section{References}

1. Berrevoet F, D'Hont F, Rogiers X, et al. Open intraperitoneal versus retromuscular mesh repair for umbilical hernias less than $3 \mathrm{~cm}$ diameter. Am J Surg 2011; 201: 85-90.

2. Christoffersen MW, Helgstrand F, Rosenberg J, et al. Lower reoperation rate for recurrence after mesh versus sutured elective repair in small umbilical and epigastric hernias. A nationwide register study. World J Surg 2013; 37: 2548-52.

3. Christoffersen MW, Helgstrand F, Rosenberg J, et al. Long-term recurrence and chronic pain after repair for small umbilical or epigastric hernias: a regional cohort study. Am J Surg 2015; 209: 725-32.

4. Helgstrand F, Rosenberg J, Kehlet $\mathrm{H}$, et al. Outcomes after emergency versus elective ventral hernia repair: a prospective nationwide study. World J Surg 2013; 37: 2273-9.

5. Armananzas L, Ruiz-Tovar J, Arroyo A, et al. Prophylactic mesh vs suture in the closure of the umbilical trocar site after laparoscopic cholecystectomy in high-risk patients for incisional hernia. A randomized clinical trial. J Am Coll Surg 2014; 218: 960-8.

6. Arroyo A, Garcia P, Perez F, et al. Randomized clinical trial comparing suture and mesh repair of umbilical hernia in adults. Br J Surg 2001; 88: 1321-3.

7. Eriksen JR, Bisgaard T, Assaadzadeh S, et al. Randomized clinical trial of fibrin sealant versus titanium tacks for mesh fixation in laparoscopic umbilical hernia repair. Br J Surg 2011; 98: 1537-45.

8. Eriksen JR, Bisgaard T, Assaadzadeh S, et al. Fibrin sealant for mesh fixation in laparoscopic umbilical hernia repair: 1-year results of a randomized controlled double-blinded study. Hernia 2013; 17: 511-4.

9. Erritzoe-Jervild L, Christoffersen MW, Helgstrand F, et al. Longterm complaints after elective repair for small umbilical or epigastric hernias. Hernia 2013; 17: 211-5.

10. Venclauskas L, Silanskaite J, Kiudelis M. Umbilical hernia: factors indicative of recurrence. Medicina (Kaunas) 2008; 44: 855-9.
11. Bencini L, Sanchez LJ, Bernini M, et al. Predictors of recurrence after laparoscopic ventral hernia repair. Surg Laparosc Endosc Percutan Tech 2009; 19: 128-32.

12. Muysoms FE, Miserez M, Berrevoet F, et al. Classification of primary and incisional abdominal wall hernias. Hernia 2009; 13: 407-14.

13. Velasco M, Gracia-Urena M, Hidalgo M, et al. Current concepts on adult umbilical hernia. Hernia 1999; 3: 233-9.

14. Lau H, Patil NG. Umbilical hernia in adults. Surg Endosc 2003; 17: 2016-20.

15. Ponten JE, Leenders BJ, Charbon JA, et al. Mesh or patch for hernia on epigastric and umbilical Sites (MORPHEUS trial): study protocol for a multi-centre patient blinded randomized controlled trial. BMC Surg 2014; 14: 33.

16. Banerjee A, Beck C, Narula VK et al. Laparoscopic ventral hernia repair: does primary repair in addition to placement of mesh decrease reccurence? Surg Endosc 2012; 26: 1264-8.

17. Aslani N, Brown CJ. Does mesh offer an advantage over tissue in the open repair of umbilical hernias? A systematic review and meta-analysis. Hernia 2010; 14: 455-62.

Received: 5.04.2017, accepted: 2.09.2017. 